\title{
Anomalous Photoelectrical Properties through Strain Engineering Based on a Single Bent InAsSb Nanowire
}

Xiaomei Yao ${ }^{\mathrm{a}, \mathrm{b}, \mathrm{c}}$, Xutao Zhang ${ }^{\mathrm{a}, \mathrm{e}}$, Qiang Sun ${ }^{\mathrm{c}}$, Dongdong Wei ${ }^{\mathrm{a}}$, Pingping Chen ${ }^{\mathrm{a}, \mathrm{b}^{*}}$, and Jin $\mathrm{Zou}^{\mathrm{c}, \mathrm{d}^{*}}$.

a State Key Laboratory of Infrared Physics, Shanghai Institute of Technical Physics Chinese Academy of Sciences, Shanghai 200083, China

${ }^{\mathrm{b}}$ University of Chinese Academy of Sciences, 19 Yuquan Road, Beijing 100049, China

${ }^{c}$ Materials Engineering, ${ }^{\mathrm{d}}$ Centre for Microscopy and Microanalysis, The University of Queensland, Brisbane, Queensland 4072, Australia.

e School of Physical Science and Technology Northwestern Polytechnical University 710129, Xi'an, China

*E-mail: ppchen@mail.sitp.ac.cn and j.zou@uq.edu.au

\section{Supporting Information:}

\section{EDS Analysis}
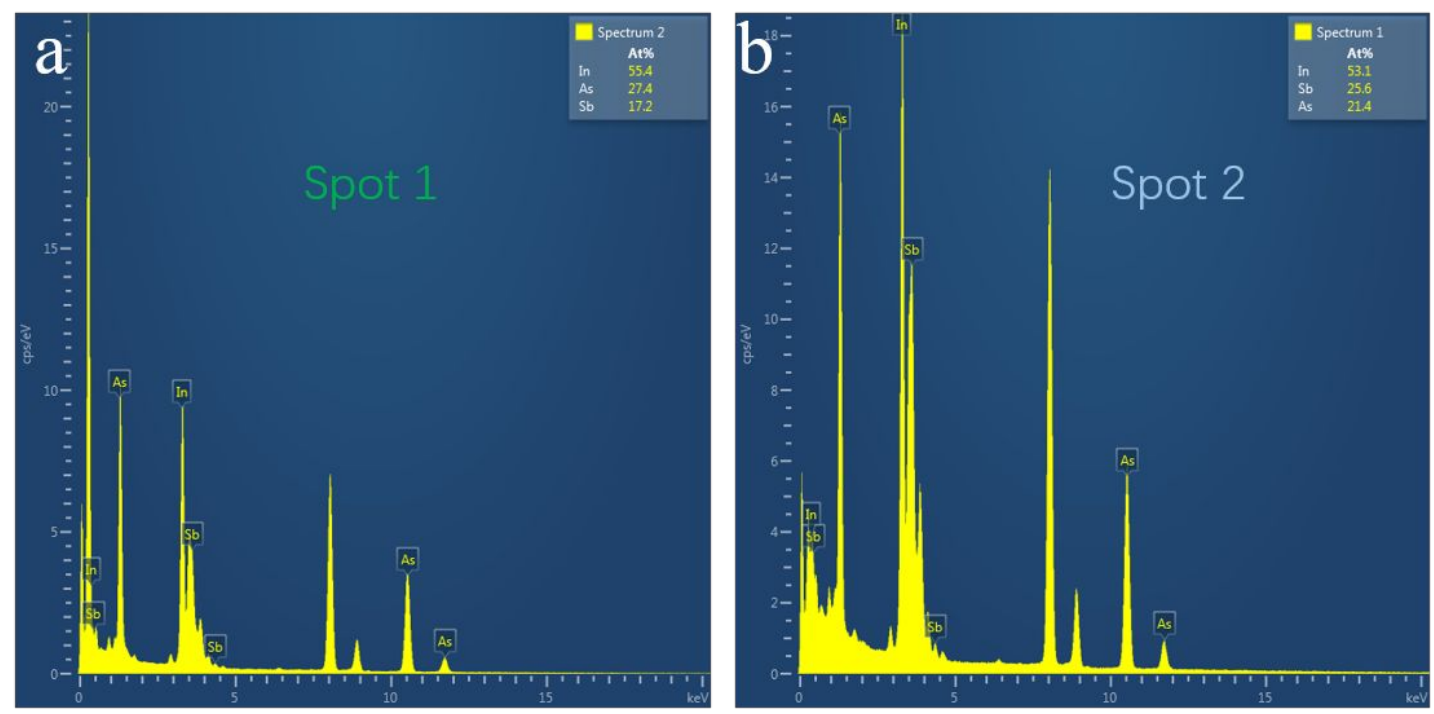

Fig. S1: (a): Original EDS data of spot 1 shown in Fig. 1(a). (b): Original EDS data of spot 2 shown in Fig. 1(a). 
The original EDS data of spot 1 and spot 2 shown in the inset of Fig. 1(a). In spot 1, the element concentration of In, As and $\mathrm{Sb}$ are 55.4\%, 27.4\%, and 17.2\%, respectively, leading to a $\mathrm{Sb}$ composition of $\sim 40 \%$. The $\mathrm{Sb}$ concentration in spot could be calculated as $\sim 45 \%$.

\section{Strain Analysis}

Fig. S2 shows the interplanar spacing of the NW in left side ((a) and (b)) and right side ((c) and (d)). Based on the statistical analysis, the plane spacing in left side is $3.85 \AA$, while in right side the value is $3.68 \AA$. The plane spacing constant for InAs and InSb are $3.49 \AA$ and $3.74 \AA$, respectively. The Sb concentration in this study is about $43 \%$, as shown in Fig. S1, indicating that the plane spacing constant of the InAsSb NW is about 3.6. Hence, the left side of the InAsSb NW is demonstrated under tensile stress, while the stress state in the right side is hard to identify. In general, the strain states due to bending structure is inhomogeneous and complicated, including tensile, compressive and shear strain. In our $\mathrm{NW}$, the tensile strain is dominated indicated by the strain mapping shown in Fig. 1(c). To simplify the problem, we only consider the tensile strain in our system. 

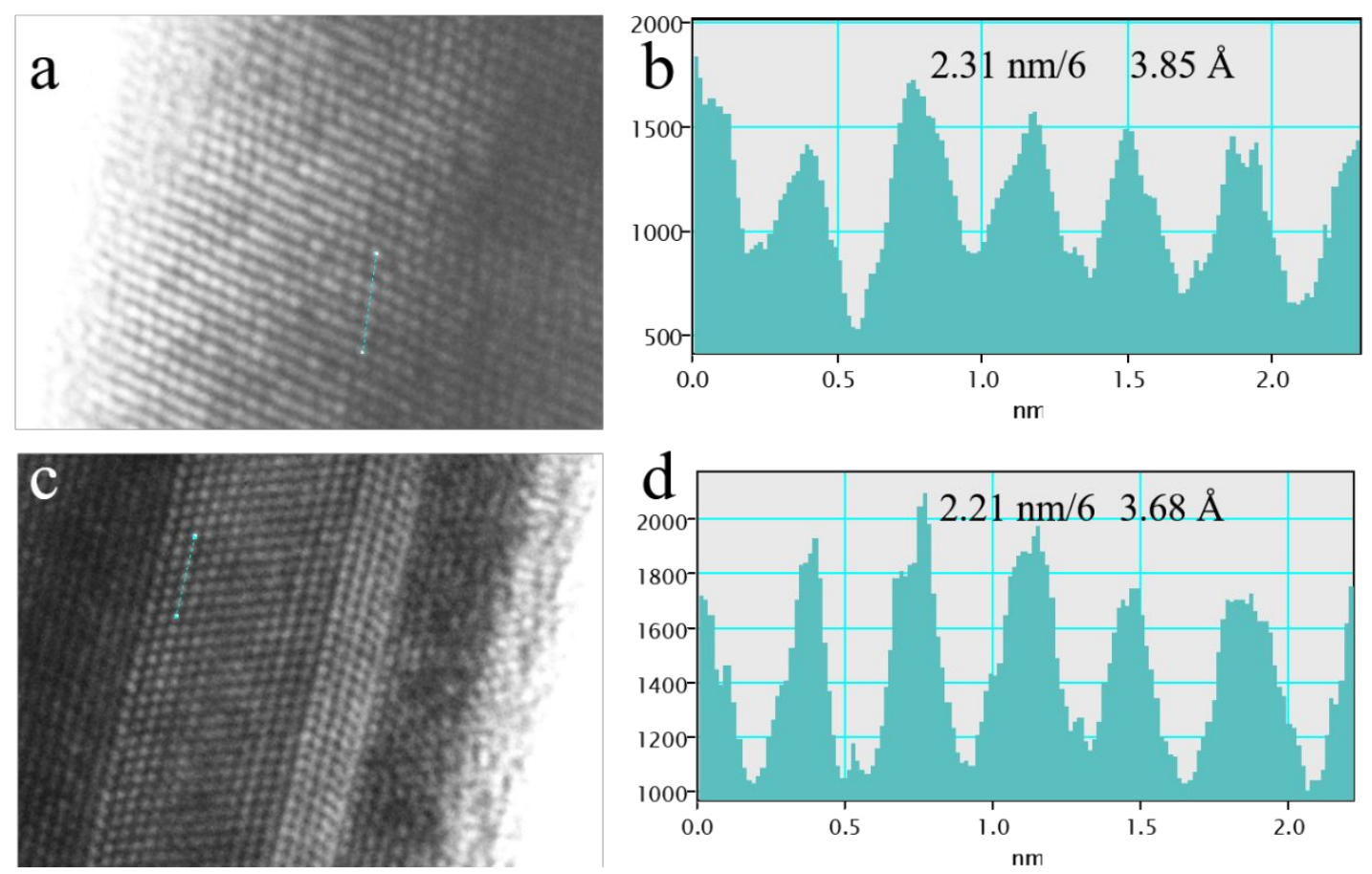

Fig. S2: The plane spacing of the bent InAsSb NW in two sides. (a) The HRTEM image of the NW in left side (tensile side). (b) The plane spacing analysis of the atoms stressed by blue lines shown in (a). (c) The HRTEM image of the NW in the right side (compressive side). (d) The plane spacing analysis of the atoms stressed by blue lines shown in (d).

The tensile strain tends to narrow the band gap, and the bottom of the conduction band would split into broad sub-bands where the free carriers mainly distribute. ${ }^{1}$ At the same time, tensile strain could reduce the effective mass of electrons and increase the lighter carrier concentration, enhancing the carrier mobility. ${ }^{2}$ 

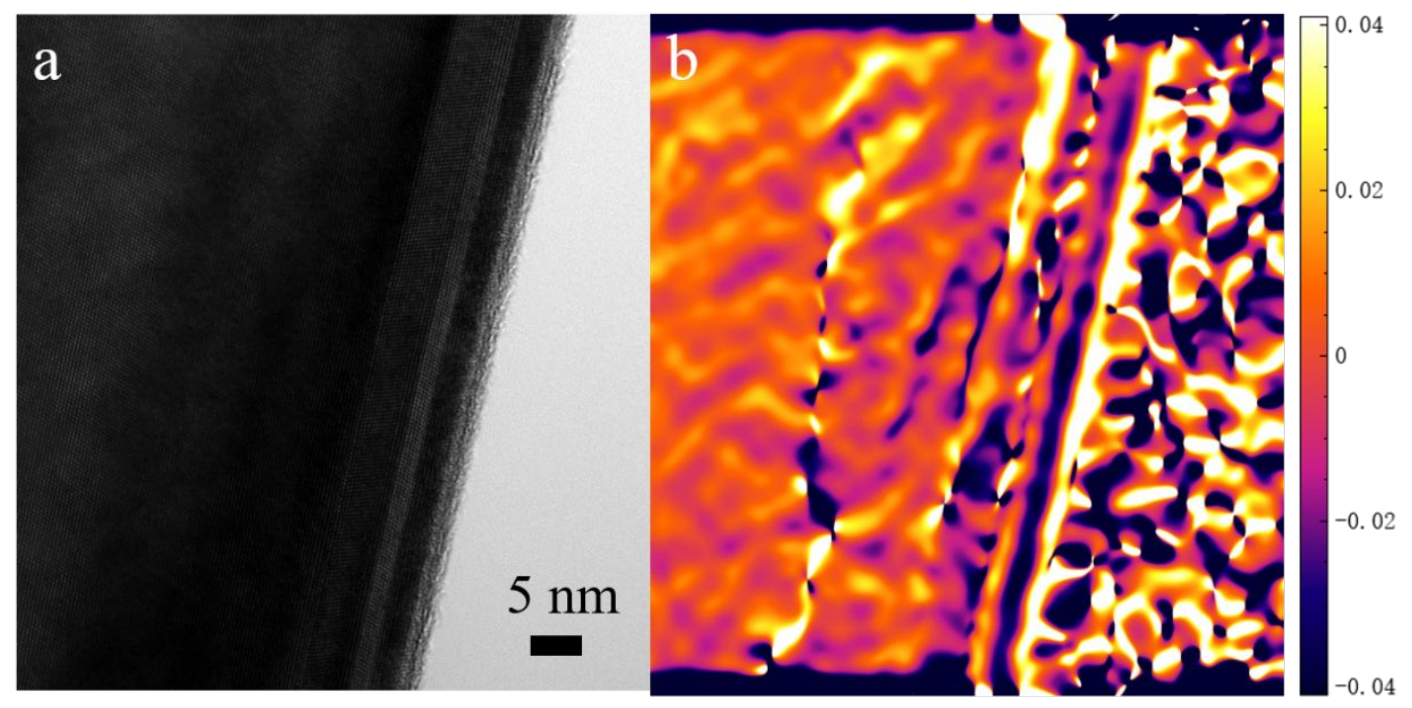

Fig. S3: The strain state of the right side of NW shown in Fig. 1(e). (a) The HRTEM image of the NW in right side. (b) Corresponding strain mapping of (a).

Fig. S3 is the strain mapping of the compressive side in the NW, indicating an obvious existence of the compressive strain.

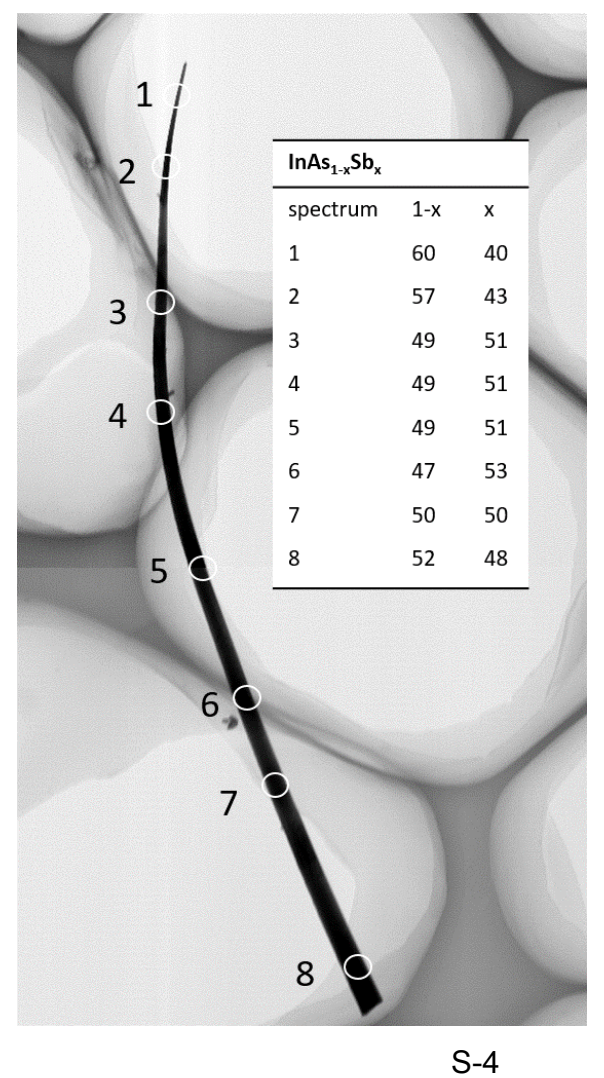


Fig. S4: The EDS result along the axial NW.

Fig. S4 shows the EDS result along the individual NW, indicating the $\mathrm{Sb}$ concentration in this sample is over $40 \%$.

\section{Electrical Measurements}

Fig. S5 shows the results of electrical measurements. The channel of the device is $3 \mu \mathrm{m}$ and the $\mathrm{Si}\left(\mathrm{p}^{+}\right) / \mathrm{SiO}_{2}(285 \mathrm{~nm})$ substrate would act as the back gate. Fig. S5(a) displays the output characteristics of the InAsSb FET at room temperature (pink line) and $77 \mathrm{~K}$ (green line), respectively. The values of conductance are about $8.9 \times 10^{6} \Omega$ and $1.5 \times 10^{6} \Omega$ at room temperature and $77 \mathrm{~K}$ respectively, indicating a five-fold decrease with the temperature decreased. Compared to the relatively straight InAsSb NWs shown in Fig. S6, the conductance value of the device is about three orders of magnitude higher. The increase conductance could be explained by the carrier density change, mass shift and scattering-rate variation attributed to the strain altered band structure according to the Drude's model. ${ }^{3}$ In the InAsSb NWs, the carrier concentration could be expressed by:

$$
n=\sqrt{N_{c} N_{v}} e^{-E g / 2 k T}
$$


where $N_{c}$ and $N_{v}$ are effect density of states in conduction and valence band, respectively, which are positively depend on the unit cell volume. Under tensile stress, the $N_{c}$ and $N_{v}$ are increase subsequently. $E_{g}$ is the energy band gap, $k$ is the Boltzmann constant and $T$ is the temperature of the device. Hence, the decrease of band gap value and increased unit cell volume would give rise to the carrier concentration in the InAsSb NWs, leading to a higher carrier concentration of the device. The carrier mobility is enhanced by the tensile strain as mentioned before. Since the conductance of the NW is determined by the carrier concentration and mobility, we can conclude the conductance is enhanced greatly with the tensile strain induced.

The transfer characteristics of the FET at room temperature and $77 \mathrm{~K}$ are shown in Fig. S5(b), indicating an N-type device. The bias voltage is $1 \mathrm{~V}$. At room temperature, the $\mathrm{ON}$-state current of the device is about $2 \mu \mathrm{A}$ while the $I_{O F F}$ is $0.2 \mathrm{nA}$, indicating a $1 \times 10^{4}$ $I_{O N} / I_{\text {OFF }}$ current ratio. By lower the test temperature to $77 \mathrm{~K}$, the $I_{O N} / I_{O F F}$ ratio would increase to about $1 \times 10^{8}$. The large value of the $I_{O N} / I_{O F F}$ ratio creates conditions for the photoelectrical modulation through the carrier regulation and low power consumption applications. For a given FET, the slope of $I_{\mathrm{ds}}-V \mathrm{~g}$ curve would be the only variate defining the mobility. As shown in Fig. S5(b), the transfer curve slope at $77 \mathrm{~K}$ is much bigger, resulting in greater mobility. ${ }^{4}$ The conductance of the device is determined by product of mobility and electron density. The conductance at $77 \mathrm{~K}$ is much smaller, indicating a 
considerable decrease in the carrier concentration. Fig. S5(c) is the Fig. S5(c)-(g) show the completed version of the time resolution curves shown in Fig. 3(c), (e) and (e) except for Fig. S5(f). In Fig. S5(f), the bias voltage is $2 \mathrm{~V}$, between $1 \mathrm{~V}$ where the bias voltage could not provide enough momentum for the photogenerated electrons and $5 \mathrm{~V}$ where enough photogenerated electrons could be trapped on the PGL and inducing NPC effect. The limited number of trapped photogenerated electrons would scatter free carriers in the channel and reduce the mobility. At the same time, the decrease carrier concentration would give rise to its mobility. Hence, the transient switch between PPC and NPC is from the critical balance of these two processes.

From the transfer curve shown in Fig.3(d), it is clear that the mobility of carriers in InAsSb NW decreases severely when it is exposed to light. Herein, it is anticipated that the NPC effect in InAsSb NWs is ascribed to the light assisted hot electron trapping which is referred as to PGL effect as well. Besides, when the light wavelength is prolonged to 1550 $\mathrm{nm}$, the transfer curve without (black line) and with (red line) light excited is shown in Fig. S5(h). The inset shows the time-resolution curve. Similar to InAs individual NWs device, the NPC effect depends tightly on the light wavelength. Essentially, the photon energy makes difference in the optical performance. Only activated by photons with enough energy, the electrons could be trapped in the surface states, inducing an NPC effect. 

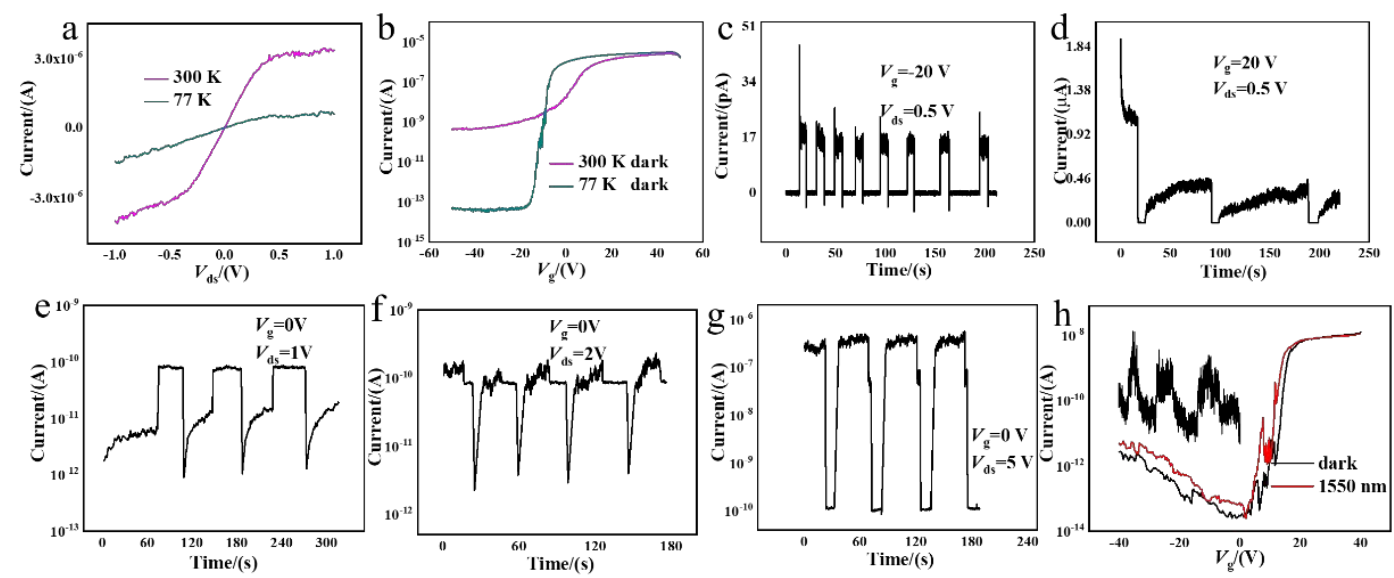

Fig. S5: (a) The output characteristics of the device at room temperature (pink line) and at $77 \mathrm{~K}$ (green line), respectively. (b) The transfer characteristics at room temperature (pink line) and at $77 \mathrm{~K}$ (green line), respectively. (c) (d) The time-resolution curves of the device when the gate voltage is $-20 \mathrm{~V}$ and $20 \mathrm{~V}$, respectively, while the bias voltage is $0.5 \mathrm{~V}$. (e) (f) (g) The time-resolution curves of the device when the bias voltage is $1 \mathrm{~V}, 2 \mathrm{~V}$ and $5 \mathrm{~V}$, respectively. (h) The transfer characteristics of the device at $77 \mathrm{~K}$ without (black line) and with $1550 \mathrm{~nm}$ light illumination (red line). The inset shows the time-resolution curve. 

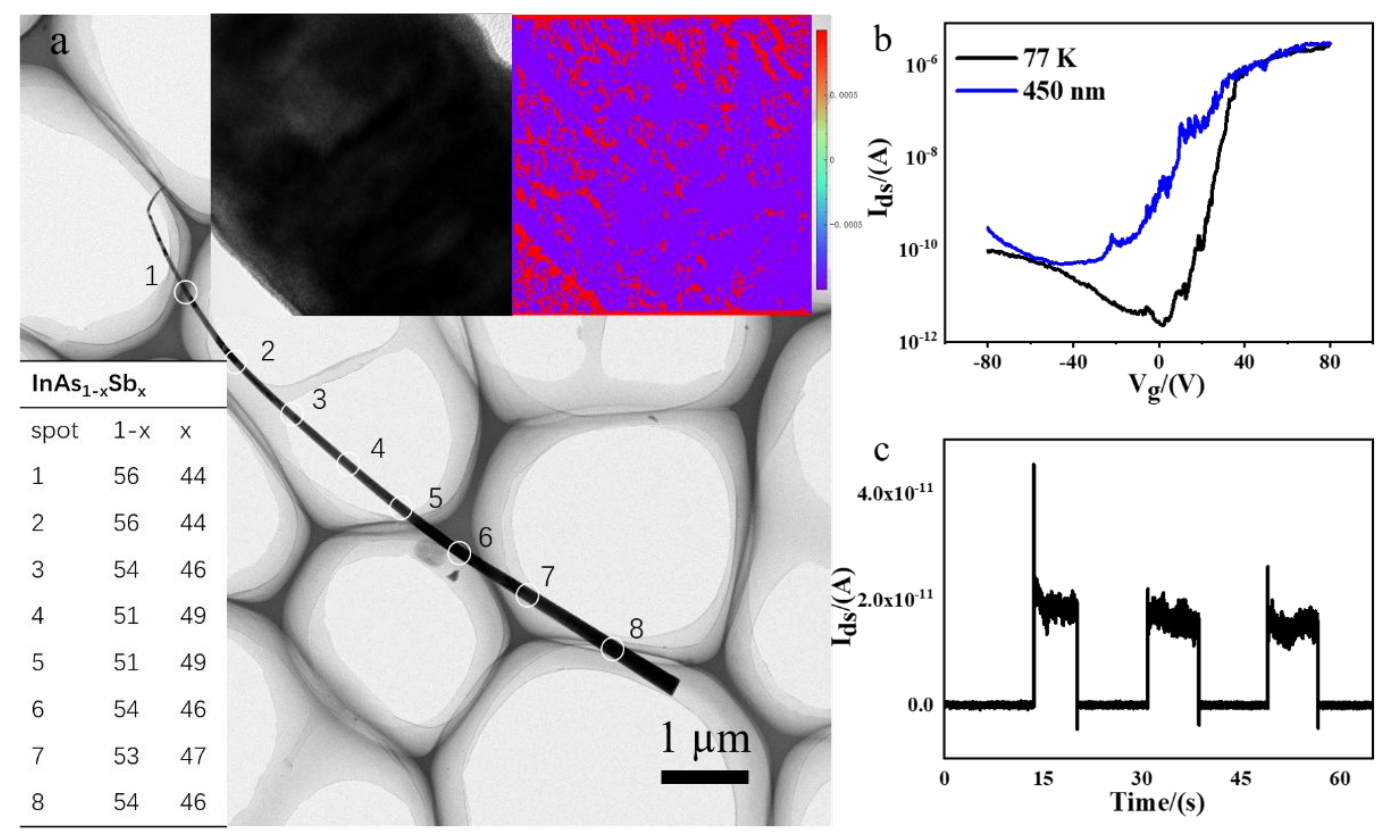

Fig. S6: The photoelectrical characteristics of the straight NW without strain. (a) The TEM image of the individual NW. The upper inset shows the HRTEM image and its corresponding strain mapping of red box in (a). The inset table is the EDS results along the axial direction of the NW.

(b) The transfer characteristics of the device without (dark line) and with (red line) $450 \mathrm{~nm}$ light illumination. (c) The time-resolution curve of the device with light emitted.

We fabricated individual InAsSb NW device with a straight NW sample without much strain. The TEM image of the individual NW is shown in Fig. S6(a), indicating tapering but straight morphology. The transfer curve and the time-resolution curves are shown in Fig. S6(b) and (c), respectively. In Fig. S6(b), the black line represents the transfer curve without light illumination, and the red line represents the transfer curve with $450 \mathrm{~nm}$ light illumination. The device shows pure PPC when the gate voltage changes. The timeresolution curve further proofs the fast PPC effect. 


\section{References}

(1) Han, X.; Jing, G.; Zhang, X.; Ma, R.; Song, X.; Xu, J.; Liao, Z.; Wang, N.; Yu, D. Bendinginduced Conductance Increase in Individual Semiconductor Nanowires and Nanobelts. Nano

Res. 2010, 2, 553-557.

(2) He, R.; Yang, P. Giant Piezoresistance Effect in Silicon Nanowires. Nat. Nanotechnol. 2006,

1.

(3) Zeng, L.; Kanne, T.; Nygård, J.; Krogstrup, P.; Jäger, W.; Olsson, E. The Effect of Bending

Deformation on Charge Transport and Electron Effective Mass of p-doped GaAs Nanowires.

Phys. Status Solidi RRL. 2019, 13.

(4) Liao, Z. M.; Hou, C.; Liu, L. P.; Yu, D. P. Temperature Dependence of Photoelectrical

Properties of Single Selenium Nanowires. Nanoscale Res. Lett. 2010, 5. 